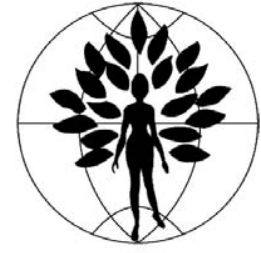

www.figo.org available at www.sciencedirect.com

www.elsevier.com/locate/ijgo

\title{
Depression, antidepressant medication, and functioning outcomes among pregnant women
}

\author{
Sheila M. Marcus *, Heather A. Flynn
}

Department of Psychiatry, University of Michigan Depression Center, University of Michigan Medical School, Ann Arbor, MI, USA

Received 29 May 2007; received in revised form 12 September 2007; accepted 13 September 2007

\author{
KEYWORDS \\ Pregnancy; \\ Depression; \\ Antidepressants; \\ Prevalence; \\ Pharmacotherapy
}

\begin{abstract}
Objectives: To describe prenatal patterns of antidepressant use and their relationship to depression in pregnancy. Methods: A total of 276 high risk women completed a structured clinical interview to investigate use of antidepressant medication. Results: Of the women interviewed, $13 \%(n=36)$ reported current use of antidepressant medication; there were no differences in depression or health functioning outcomes between women taking antidepressants and those not using them. Conclusions: Monitoring the depressive symptoms of women using pharmacotherapy during pregnancy is important to optimize their treatment.

(c) 2007 International Federation of Gynecology and Obstetrics. Published by Elsevier Ireland Ltd. All rights reserved.
\end{abstract}

\section{Introduction}

Major depressive disorder (MDD) and elevated depressive symptoms are common in pregnancy [1] and both have been linked to pregnancy and birth complications, as well as poor neonatal outcomes. In one study, the investigators found a significantly higher risk of low and very low birth weight and preterm neonates in women with a psychiatric diagnosis [2]. Other studies have linked prenatal depression to elevated fetal activity and delayed prenatal growth [3], and postnatal depression to adverse neonatal outcomes [4,5]. Recent reports have also suggested that treatments for depression around the time of pregnancy are underutilized [6]. Moreover,

\footnotetext{
* Corresponding author. Department of Psychiatry, University of Michigan Medical School, 4250 Plymouth Road Box 5766, Ann Arbor, MI 48109-5766, USA. Tel.: +1 7640245; fax: +1 7349368907.

E-mail address: smmarcus@umich.edu (S.M. Marcus).
}

Cohen et al. [7] showed that up to $80 \%$ of women may relapse when their medication is discontinued during pregnancy.

There is a great deal of controversy in the literature regarding the use of antidepressants during pregnancy. The majority of early studies examining the use of antidepressants during pregnancy suggest that the tricyclic antidepressants and selective serotonin reuptake inhibitors (SSRIs) are unlikely to contribute to major congenital anomalies above the baseline risk of $1 \%-3 \%$ seen in the general population [8-13]. However, some studies do suggest that first trimester exposure to SSRIs may contribute to preterm delivery, restricted fetal growth, and minor malformations [14-16]. Additionally, there have been several reports suggesting that prenatal exposure to SSRIs may be associated with neonatal withdrawal including irritability, increased tone, increased crying, and sleep and appetite dysregulation [17]. A recent report by GlaxoSmithKline [18] suggesting that infants exposed to paroxetine during the first trimester had an increased risk of congenital malformations ( $4 \%$ vs $2 \%$ in the general

0020-7292/\$ - see front matter @ $₫ 2007$ International Federation of Gynecology and Obstetrics. Published by Elsevier Ireland Ltd. All rights reserved. 
population), particularly cardiovascular anomalies (3\% vs $1 \%$ in the general population), resulted in the reclassification of paroxetine as a category $\mathrm{D}$ agent during pregnancy. Finally, Chambers et al. [14] suggest that persistent pulmonary hypertension of the newborn may be associated with late trimester use of SSRIs. In these studies there was no control for maternal illness or symptom severity. As such, the relative contribution of undertreated maternal depressive symptoms and illness severity with use of SSRIs was not examined. Only one study compared control (depression plus no fluoxetine) and treated (depression plus fluoxetine) and found no effect of fluoxetine exposure on birth weight, gestational age, or Neonatal Intensive Care Unit (NICU) admissions [19].

The purpose of the present study was to describe the rates and patterns of the use of common antidepressants during pregnancy, and to examine the relationship of different usage patterns of antidepressants to depressive symptoms and health functioning in a cohort of depressed, pregnant women identified in obstetrics settings.

\section{Methods}

As part of a larger pregnancy screening project, women were screened for depressive symptoms (mean $24 \pm 10.4$ gestational weeks) when seeking prenatal care at 5 University of Michigan affiliated obstetrics clinics. The clinics serve patients with managed care insurance plans (70.6\%), Medicare $(7.9 \%)$, Medicaid $(14.4 \%)$, and no or unknown insurance $(7.1 \%)$. The mean age of participants was $28.6 \pm 6$ years. Most were married (74\%); others had a live-in partner $(10 \%)$, were separated $(1.3 \%)$, divorced $(1.8 \%)$, widowed $(0.1 \%)$, or never married $(12.8 \%)$. The racial distribution of the sample reflected that of southeast lower Michigan as follows: $73 \%$ white, $13 \%$ African American, 9.3\% Asian, 2.4\% Hispanic, 0.7\% Native American, and 1.6\% other race. Full details about screening procedures have been described in detail elsewhere [6]. A total of 276 (16\% of those screened) women were eligible and completed prenatal interviews based on a Center for Epidemiologic Studies Depression Scale (CES-D) score of 16 or greater, self-reported major depression in the past 6 months, or discontinuation of antidepressant medications due to conception. Confidentiality was maintained, informed consent obtained, and all study procedures were approved by the Institutional Review Board of the University of Michigan Medical School.

Depressive symptomatology was measured using the Center for Epidemiological Studies Depression Scale (CES-D). The CES-D is used widely as a depression screening instrument in nonclinical populations, with good correlation with the Beck Depression Inventory $(r=0.87)$ [20]. The standard cutoff score of 16 was used to determine significantly elevated depressive symptomatology. The Structured Clinical Interview for DSM-IV (SCID) interview was administered to assess presence and severity of current and past psychiatric disorders. As reported previously [6], $17 \%(46 / 276)$ of the sample met criteria for current MDD based on SCID interview, and an additional $23 \%$ met criteria for a past history of MDD $(n=63)$. The Beck Depression Inventory-II was also administered as a repeated measure to assess severity of symptoms of depression. Health Functioning was assessed using the Medical Outcome Study (MOS) Short Form Survey (SF-36) [21], which has been used as an indicator of functional status changes during pregnancy $[4,5]$, and measures 8 domains: general health, physical functioning, physical role functioning, bodily pain, vitality, mental health, emotional role functioning, and social functioning.

Data analysis first described rates of antidepressant medication use among the sample. Mean differences on BDI-II and SF36 scores were examined using $t$ test, with $P=0.05$ considered statistically significant. Means, standard deviations, and ranges of each antidepressant medication dosage were also computed.

\section{Results}

Of all women completing the prenatal interview, $13 \%(n=36)$ reported current use of antidepressant medication. As previously reported [6], an additional 16 (6\%) were receiving any other form of treatment, and a minority of women who met criteria for current MDD $(19 \%, n=9)$ were taking antidepressant medication. No differences were found in rates of medication use between MDD and the rest of the sample (i.e., women with risk for depression as defined above but no current MDD). As assessed during pregnancy, no significant differences in BDI-II scores or SF-36 health functioning indices were found between women with MDD currently taking antidepressants and those not taking antidepressants (mean BDI-II with medications $24.9 \pm 7.4$ vs mean BDI-II without medication $28.1 \pm 9.1$ ). Similarly, when examining the total high risk sample (regardless of MDD status), no differences between women taking antidepressants and those not taking medications were found on BDI-II scores (no antidepressants mean $16.1 \pm 9.4$ vs taking antidepressants mean $18.9 \pm 9.4$ ). No differences between women taking and not taking antidepressants were found in the any of the SF-36 scales, with the exception of emotional role functioning. Women using antidepressants showed significantly worse emotional role functioning (which indicates the extent to which work and other important activities were impacted by psychiatric symptoms) than those not taking antidepressants $(t[1,273]=$ 4.1, $P<0.05)$.

Given that antidepressant medication use was not associated with symptom differences compared with the nonmedicated group, we were interested in the duration and dosage of the medications used by women in our sample, as well as the consistency of use. Table 1 shows the mean dosage, duration of use, as well as the ranges of each reported antidepressant medication. Among the interviewed sample of women who reported any antidepressant medication use in

Table 1 Antidepressant medication use reported during pregnancy: mean dosage and duration of use $(n=36)^{\mathrm{a}}$

\begin{tabular}{lrcc}
\hline Drug name & No. ${ }^{\mathrm{b}}$ & Dosage, mg & $\begin{array}{l}\text { Duration of use } \\
\text { in months }\end{array}$ \\
\hline Fluoxetine & 12 & $23.3(10-40)$ & $18(1-108)$ \\
Sertraline & 8 & $68.7(50-100)$ & $10.2(1-48)$ \\
Paroxetine & 5 & $30(10-60)$ & $11(<1-24)$ \\
Bupropion & 3 & $166.6(100-200)$ & $14.5(<1-36)$ \\
Citalopram & 3 & $20(--)$ & $4(3-5)$ \\
Venlafaxine & 1 & $175(--)$ & $20(--)$ \\
\hline
\end{tabular}

${ }^{a}$ Values are given as mean (range).

${ }^{\mathrm{b}}$ The number reported in the table is lower than the total number of women reporting antidepressant use due to missing dosage information. 
the 2 years prior to pregnancy, 3 categories regarding continuity of use emerged: 1 group who reported discontinuing use of antidepressants as a result of pregnancy (41\%), 1 group who reported discontinuing use due to pregnancy and then resumed use at some point during pregnancy (21\%), and 1 group that continued antidepressant use into pregnancy (38\%). No significant differences in mean BDI-II scores or SF-36 health functioning indices were found among the 3 groups, but all 3 groups showed mean elevated BDI-II scores $(18.7 \pm 11 ; 21.5 \pm 9.4 ; 17.7 \pm 9.5$, respectively $)$.

\section{Discussion}

The present study compared depression and health functioning measures in pregnant women recruited from obstetrics settings taking antidepressant medication with women not taking antidepressants. The study found that the majority of women using antidepressant medication continued to experience mood symptoms despite their use, suggesting suboptimal treatment. The present study adds to our previous work in this area by examining depressive symptomatology (as measured by the BDI-II) and health functioning in women with and without a SCID diagnosis of MDD using antidepressant medication during pregnancy, as well as detailed information on dosing and duration of treatment within this symptomatic group based on interview data. The findings are similar to those reported in a previous screening study [22] in which women using and not using antidepressant medication showed similar elevations in CES-D scores. However, that previous cohort of women was comprised of a general prenatal care seeking sample, and information on MDD status and on dosing and duration of medication treatment was not obtained.

The group of women investigated in this study was a high risk sample for depression, with $17 \%$ meeting current criteria for MDD by diagnostic interview and an additional 24\% for past history of MDD, the strongest risk factor for subsequent depression. The majority of women studied continued to show elevated mood symptoms as measured by the BDI-II during their pregnancy and there were no differences in symptom severity or of health functioning between those taking medication compared with women not taking medication. Although most women reported daily use for at least 8 weeks, the mean dosage of both venlafaxine and bupropion was below the dosage that commonly permits full remission of symptoms. Likewise in clinical practice, SSRI doses frequently exceed the mean dosage of fluoxetine, paroxetine and citalopram observed in this study. Furthermore, studies with the tricyclic antidepressants revealed that as maternal plasma volumes increased, antidepressant blood levels fall with consequent re-emergence of mood symptoms during later pregnancy $[23,24]$. While there are no published data on optimal antidepressant dosing during pregnancy and no studies specifically discussing efficacy of antidepressants during pregnancy, the American Psychiatric Association Treatment Recommendations for Patients with Major Depressive Disorder recommends the following daily dosages: $20-60 \mathrm{mg}$ of citalopram, $20-80 \mathrm{mg}$ of fluoxetine, $20-60 \mathrm{mg}$ of paroxetine, $150-450 \mathrm{mg}$ of bupropion, and $150-375 \mathrm{mg}$ of venlafaxine. Among these women $(n=254), 70 \%$ reported some form of counseling or therapy.
The service implications for these findings are considerable and suggest that women merit much closer monitoring for depressive symptoms during pregnancy. In our sample, most women were not treated, and many remained symptomatic despite use of pharmacotherapy. It is critical that physicians treating pregnant women monitor symptoms so that women can be properly referred for treatment when indicated and pharmacotherapy can be optimized when women remain symptomatic. Many studies [25] document improvement in cognitive outcomes of infants when their mothers are treated for depression in pregnancy.

One important methodological consideration of this study was that only women who reported some risk of depression (primarily defined as elevated score on the depression screening measure) were included in the study. Therefore, we may not have captured asymptomatic women taking antidepressants. Recruitment analyses showed that only $11 \%$ of women who had been taking antidepressants in the 2-year period prior to screening were not eligible to be interviewed based on our depression risk criteria, suggesting that the majority (89\%) of all users of antidepressants were captured in this study. Nonetheless, future studies should compare depression outcomes among medication use groups unselected for depression risk. Another limitation of the study is that, although women reported dosage, duration and daily use of the medications, adherence and pharmacy data were not obtained. It is noted that a randomized controlled trial would be necessary to fully study the effectiveness of medications during pregnancy, however, most internal review boards disallow such trials using antidepressant medication during pregnancy. Moreover, it is acknowledged that depressive symptomatology may have been impacted by virtue of study participation.

For both pregnant and nonpregnant women alike, remission of symptoms is the goal. Simultaneous exposure to both medication and symptoms of depression during pregnancy may put the fetus at unnecessary risk. Furthermore, current studies exploring the relative risk of pharmacotherapy during pregnancy may be reporting on both the risks of pharmacotherapy and undertreated depression, if the sample population is similar to this community sample. It underscores the clinical importance of monitoring mood symptoms when using pharmacotherapy during pregnancy and its consideration when reporting the potential medication effects on the neonate.

\section{Acknowledgement}

Initial funding from the University of Michigan Faculty Group Practice made this study possible.

\section{References}

[1] Marcus SM, Flynn HA, Blow F, Barry KL. Depressive symptoms among pregnant women screened in obstetrics settings. J Women's Health (Larchmt) 2003;12:373-80.

[2] Kelly RH, Russo J, Holt VL, Danielsen BH, Zatzick DF, Walter E, et al. Psychiatric and substance use disorders as risk factors for low birth weight and preterm delivery. Obstet Gynecol 2002;100: 297-304. 
[3] Field T, Diego M, Hernandez-Reif M. Prenatal depression effects on the fetus and newborn: a review. Infant Behav Dev 2006;29: 445-55.

[4] McKee MD, Cunningham M, Jankowski K, Zayas L. Healthrelated functional status in pregnancy: relationship to depression and social support in a multi-ethnic population. Obstet Gynecol 2001;97:988-93.

[5] Hueston WJ, Kasik-Miller S. Changes in functional health status during normal pregnancy. J Fam Pract 1998;47:209-12.

[6] Flynn HA, Blow FC, Marcus SM. Rates and predictors of depression treatment among pregnant women in hospital-affiliated obstetrics practices. Gen Hosp Psych 2006;28:289-95.

[7] Cohen LS, Altshuler LL, Harlow BL, Nonacs R, Newport DJ, Viguera AC, et al. Relapse of major depression during pregnancy in women who maintain or discontinue antidepressant treatment. JAMA 2006;295:499-507.

[8] Altshuler LL, Cohen L, Szuba MP, Burt VK, Gitlin M, Mintz J. Pharmacologic management of psychiatric illness during pregnancy: dilemmas and guidelines. Am J Psychiatr 1996;153: 592-606.

[9] Addis A, Koren G. Safety of fluoxetine during the first trimester of pregnancy: a meta-analytical review of epidemiological studies. Psychol Med 2000;30:89-94.

[10] Cohen LS, Heller VL, Bailey JW, Grush L, Ablon JS, Bouffard SM. Birth outcomes following prenatal exposure to fluoxetine. Biol Psychiatry 2000;48:996-1000.

[11] Goldstein DJ. Effects of third trimester fluoxetine exposure on the newborn. J Clin Psychopharmacol 1995;15:417-20.

[12] Nulman I, Koren G. The safety of fluoxetine during pregnancy and lactation. Teratology 1996;53:304-8.

[13] Ericson A, Kallen B, Wiholm B. Delivery outcome after the use of antidepressants in early pregnancy. Eur J Clin Pharmacol 1999;55:503-8.

[14] Chambers CD, Hernandez-Diaz S, Van Marter LJ, Werler MM, Louik C, Jones KL, et al. Selective serotonin-reuptake inhibitors and risk of persistent pulmonary hypertension of the newborn. New Engl J Med 2006;354:579-87.

[15] Hendrick V, Smith LM, Suri R, Hwang S, Haynes D, Altshuler L. Birth outcomes after prenatal exposure to antidepressant medication. Am J Obstet Gynecol 2003;188:812-5.

[16] Kallen B. Neonate characteristics after maternal use of antidepressants in late pregnancy. Arch Pediatr Adolesc Med 2004;158: 312-6.

[17] Nordeng H, Lindemann R, Perminov KV, Reikvam A. Neonatal withdrawal syndrome after in utero exposure to selective serotonin reuptake inhibitors. Acta Paediatr 2001;90:288-91.

[18] GlaxoSmithKline. Study EPIP083 (Preliminary report) 2005. http://ctr.gsk.co.uk/Summary/paroxetine/studylist.asp.

[19] Suri R, Altshuler L, Hendrick V, Rasgon N, Lee E, Mintz J. The impact of depression and fluoxetine treatment on obstetrical outcome. Arch Women Ment Health 2004;7:192-200.

[20] Santor DA, Zuroff DC, Ramsay JO, Cervantes P, Palacios J. Examining scale discriminability in the BDI and CES-D as a function of depressive severity. Psychol Assess 1995;7:131-9.

[21] Hayes RD, Sherbourne CD, Mazel RM. The RAND 36-Item health survey 1.0. Health Econ 1993;2:217-27.

[22] Marcus SM, Flynn HA, Blow F, Barry K. A screening study of antidepressant treatment rates and mood symptoms in pregnancy. Arch Women Ment Health 2005;8:25-7.

[23] Altshuler LL, Hendrick VC. Pregnancy and psychotropic medication: changes in blood levels. J Clin Psychopharmacol 1996; Feb;16:78-80.

[24] Wisner KL, Perel JM, Wheeler SB. Tricyclic dosage requirements across pregnancy. Am J Psychiatry 1993;150:1541-52.

[25] Nulman I, Rovet J, Stewart DE, Wolpin J, Pace-Asciak P, Shuhaiber S, et al. Child development following exposure to tricyclic antidepressants or fluoxetine throughout fetal life: a prospective, controlled study. Am J Psychiatry 2002;159:1889-95. 